\title{
Erratum to: c-Myc-regulated long non-coding RNA H19 indicates a poor prognosis and affects cell proliferation in non-small-cell lung cancer
}

\author{
Erbao Zhang ${ }^{1} \cdot$ Wei $\mathrm{Li}^{2} \cdot$ Dandan Yin $^{3} \cdot$ Wei De ${ }^{1} \cdot$ Liangjun Zhu ${ }^{4} \cdot$ Sanyuan Sun ${ }^{5}$. \\ Liang $\operatorname{Han}^{5}$
}

Published online: 11 December 2015

(C) International Society of Oncology and BioMarkers (ISOBM) 2015

\section{Erratum to: Tumor Biol}

DOI 10.1007/s13277-015-4185-5

The original article contained an error.

The additional author and its corresponding affiliation are missing.

The corrected author group is shown above and its corresponding affiliation is shown below.

The online version of the original article can be found at http://dx.doi.org/ 10.1007/s13277-015-4185-5.

\footnotetext{
Liang Han

lianghan_xuzhou@163.com

$\triangle$ Sanyuan Sun

sanyuan_sun@sina.com

$\triangle$ Liangjun Zhu

liangjunzhu_njmu@163.com
}

1 Department of Biochemistry and Molecular Biology, Nanjing Medical University, Nanjing, Jiangsu, People's Republic of China

2 Department of Oncology, First Affiliated Hospital of Nanjing Medical University, Nanjing, Jiangsu, People's Republic of China

3 Central Laboratory, The Second Affiliated Hospital of Southeast University, Nanjing, Jiangsu, People's Republic of China

4 Department of Oncology, Nanjing Medical University Affiliated Cancer Hospital, Cancer Institute of Jiangsu Province, Baiziting 42, Nanjing 210009, China

5 Department of Oncology, Xuzhou Central Hospital, Affiliated Xuzhou Hospital, College of Medicine, Southeast University, Xuzhou, Jiangsu, People's Republic of China 